To be reported at $17^{\text {th }}$ Int. Conference on High Magnetic Fields, Würzburg, Germany, July 30 - August 4, 2006

\title{
Many-body Effects in Landau Levels: Non-commutative Geometry and Squeezed Correlated States
}

\author{
Alexander B. Dzyubenkd* \\ Department of Physics, California State University at Bakersfield, Bakersfield, CA 93311, USA and \\ General Physics Institute, Russian Academy of Sciences, Vavilova 38, Moscow 119991, Russia
}

(Dated: July 8, 2018)

\begin{abstract}
We discuss symmetry-driven squeezing and coherent states of few-particle systems in magnetic fields. An operator approach using canonical transformations and the $S U(1,1)$ algebras is developed for considering Coulomb correlations in the lowest Landau levels.
\end{abstract}

Keywords: High Magnetic Fields; Coulomb Correlations; Coherent States.

\section{INTRODUCTION}

Coulomb interactions in strong magnetic fields are relevant in the various physical contexts such as, for example, the behavior of ions in atmospheres of neutron stars, electrons and holes in semiconductors, and charged quasiparticles in the Fractional Quantum Hall Effect regime. One of the general aspects of the problem are considerations of the relevant symmetries. In this work, we discuss an operator method that allows one to maintain both axial and magnetic translations geometric symmetries in two-dimensional (2D) systems in Landau Levels (LL). We also establish a connection with the dynamical $S U(1,1)$ symmetry.

\section{CHARGED $e-h$ SYSTEMS}

Let us consider a $2 \mathrm{D}$ system of two oppositely charged particles $-q_{1}<0$ and $q_{2}>0$, which we will denote as an "electron" and a "hole", respectively. The total charge is negative, $-Q=q_{2}-q_{1}<0$. The operator of magnetic translations (MT) is of the form $\hat{\mathbf{K}}=$ $-i \hbar \boldsymbol{\nabla}_{1}+-i \hbar \boldsymbol{\nabla}_{2}-\mathbf{B} \times\left(q_{1} \mathbf{r}_{1}-q_{2} \mathbf{r}_{2}\right) / 2 c$, where the symmetric gauge is used 1.2 The MT group is non-commutative, and the dimensionless MT operator $\hat{\mathbf{k}}=\hat{\mathbf{K}} L_{B} / \hbar$ has canonically conjugate components, $\left[\hat{k}_{x}, \hat{k}_{y}\right]=i$, where $L_{B} \equiv \sqrt{\hbar c / Q B}$ is the effective magnetic length. This allows one to introduce a pair of Bose ladder operators for the whole system,, $8 \tilde{B}_{e}^{\dagger}=-i \hat{k}_{-} / \sqrt{2}$ and $\tilde{B}_{e}=i \hat{k}_{+} / \sqrt{2}$ such that $\left[\tilde{B}_{e}, \tilde{B}_{e}^{\dagger}\right]=1$, here $\hat{k}_{ \pm}=\hat{k}_{x} \pm i \hat{k}_{y}$. In terms of intra-LL operators of individual particles, we have ${ }^{2}$

$$
\begin{gathered}
\tilde{B}_{e}^{\dagger}=-\frac{i \hat{k}_{-}}{\sqrt{2}}=u B_{e}^{\dagger}-v B_{h} \\
u=\sqrt{\frac{q_{1}}{Q}}, \quad v=\sqrt{\frac{q_{2}}{Q}} .
\end{gathered}
$$

The second linearly independent annihilation operator is $\tilde{B}_{h}=u B_{h}-v B_{e}^{\dagger}$ so that we have two pairs of Bose ladder operators: $\left[\tilde{B}_{h}, \tilde{B}_{h}^{\dagger}\right]=\left[\tilde{B}_{e}, \tilde{B}_{e}^{\dagger}\right]=1,\left[\tilde{B}_{h}, \tilde{B}_{e}^{\dagger}\right]=0$, and $\left[\tilde{B}_{h}, \tilde{B}_{e}\right]=0$. This is in fact Bogoliubov canonical transformation

$$
\begin{aligned}
\left(\begin{array}{c}
\tilde{B}_{e}^{\dagger} \\
\tilde{B}_{h}
\end{array}\right) & =\left(\begin{array}{c}
\tilde{S} B_{e}^{\dagger} \tilde{S}^{\dagger} \\
\tilde{S} B_{h} \tilde{S}^{\dagger}
\end{array}\right)=\hat{U}\left(\begin{array}{c}
B_{e}^{\dagger} \\
B_{h}
\end{array}\right), \\
\hat{U} & \equiv\left(\begin{array}{rr}
\cosh \Theta & -\sinh \Theta \\
-\sinh \Theta & \cosh \Theta
\end{array}\right)
\end{aligned}
$$

performed by the unitary operator $\tilde{S}=\exp (\Theta \tilde{\mathcal{L}})$ with the generator $\tilde{\mathcal{L}}=B_{h}^{\dagger} B_{e}^{\dagger}-B_{e} B_{h}$; here $\Theta$ is the transformation parameter with $u=\cosh \Theta, v=\sinh \Theta$. This transformation introduces new quasiparticles with coordinates

$$
\mathbf{R}_{1}=\frac{q_{1} \mathbf{r}_{1}-q_{2} \mathbf{r}_{2}}{Q} \quad, \quad \mathbf{R}_{2}=\frac{\sqrt{q_{1} q_{2}}}{Q}\left(\mathbf{r}_{2}-\mathbf{r}_{1}\right)
$$

in which the transformed operators assume the standard forms ${ }^{2}$

$$
\begin{aligned}
& \tilde{B}_{e}^{\dagger}=\frac{1}{\sqrt{2}}\left(\frac{Z_{1}^{*}}{2 L_{B}}-2 L_{B} \frac{\partial}{\partial Z_{1}}\right) \\
& \tilde{B}_{h}^{\dagger}=\frac{1}{\sqrt{2}}\left(\frac{Z_{2}}{2 L_{B}}-2 L_{B} \frac{\partial}{\partial Z_{2}^{*}}\right)
\end{aligned}
$$

where the $2 \mathrm{D}$ complex variables $Z_{i}=X_{i}+i Y_{i}$ are used. Note that the interaction potential $U_{\text {int }}=U\left(\mathbf{r}_{1}-\mathbf{r}_{2}\right)$ does not depend on $\mathbf{R}_{1}$. The MT operator is diagonal in the new representation, $\mathbf{k}^{2}=2 \tilde{B}_{e}^{\dagger} \tilde{B}_{e}+1$. It has the discrete spectrum $2 k+1$, where the oscillator quantum numbers $k=0,1,2, \ldots$ determine the position of a guiding center of a charged system in $\mathbf{B}^{1.2} \mathrm{~A}$ complete basis of states in zero LL compatible with both axial and translational symmetries is given by

$$
\frac{\tilde{B}_{e}^{\dagger k} \tilde{B}_{h}^{\dagger m}}{\sqrt{k ! m !}}|\tilde{0}\rangle \equiv|\widetilde{k m}\rangle
$$

where $|\tilde{0}\rangle=\tilde{S}|0\rangle$ is the transformed vacuum and state $|\widetilde{k m}\rangle$ has total angular momentum projection $M_{z}=$ $m-k$. The energy spectrum is degenerate with respect to $k$. Therefore, it is sufficient to consider only the states with $k=0$ from (8); we denote such states as $|\tilde{m}\rangle$. The above procedure removes one degree of freedom and corresponds to a possible partial separation of variables in magnetic fields. 


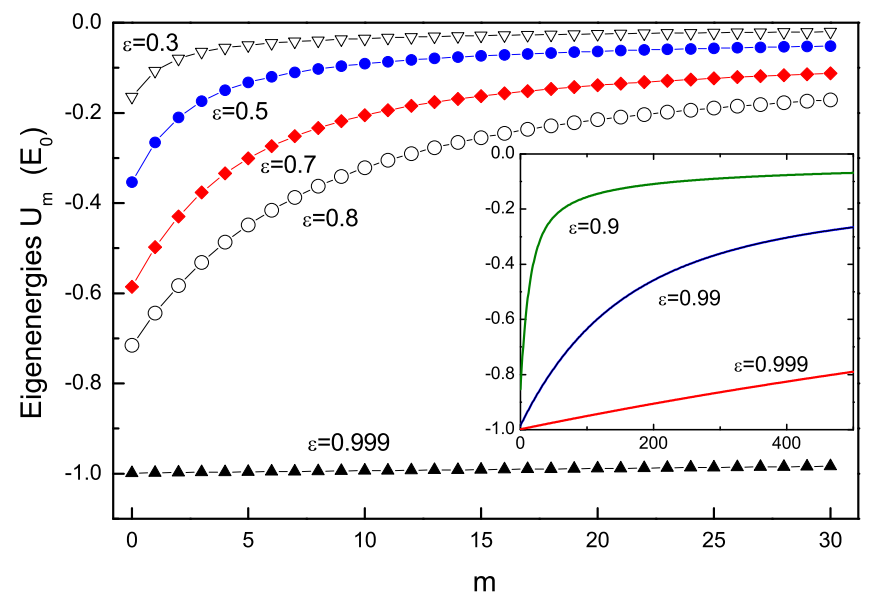

FIG. 1: Eigenergies (9) of two interacting charges $-q_{1}<0$ and $q_{2}>0$ in zero LL for several values of parameter $\epsilon=$ $\left(q_{2} / q_{1}\right)^{1 / 2}$.

For, e.g., the Coulomb interaction $U_{\text {int }}=-q_{1} q_{2} / \mid \mathbf{r}_{1}-$ $\mathbf{r}_{2} \mid$, the eigenergies in the lowest LL can be calculated analytically as expectation values:

$$
\begin{aligned}
U_{m} & =\left\langle\tilde{m}\left|U_{\mathrm{int}}\right| \tilde{m}\right\rangle \\
& =-E_{0}\left(\frac{q_{2}}{q_{1}}\right)^{m+\frac{3}{2}} \sum_{k=0}^{m} C_{m}^{k} \frac{\Gamma\left(k+\frac{1}{2}\right)}{\sqrt{\pi} k !}\left(\frac{q_{1}-q_{2}}{q_{2}}\right)^{k},
\end{aligned}
$$

where $E_{0}=\sqrt{\frac{\pi}{2}} \frac{q_{1}^{2}}{l_{B 1}}, l_{B i}=\left(\hbar c / q_{i} B\right)^{1 / 2}$ are magnetic lengths. Eigenenergies (9) are shown in Fig. 1 for several values of parameter $\epsilon=\left(q_{2} / q_{1}\right)^{1 / 2}<1$. The spectra are completely discrete. However, in the limit $q_{2} \rightarrow q_{1}$ $\left(\epsilon \rightarrow 1^{-0}\right)$ the spectra become quasicontinuous and fill in the $2 \mathrm{D}$ neutral magnetoexciton band of width $E_{0}, \frac{3}{3}$

In the terminology of quantum optics, the transformed vacuum $|\tilde{0}\rangle=\tilde{S}|0\rangle$ is a two-mode squeezed state $\stackrel{\underline{4}}{\underline{n}}$ For particles in a magnetic field squeezing has a direct geometrical meaning $\stackrel{2}{=}$ Indeed, in the coordinate representation we have

$$
\left\langle\mathbf{r}_{1}, \mathbf{r}_{2} \mid \tilde{0}\right\rangle=\frac{\sqrt{1-\epsilon^{2}}}{2 \pi l_{B 1} l_{B 2}} \exp \left(-\frac{\mathbf{r}_{1}^{2}}{4 l_{B 1}^{2}}-\frac{\mathbf{r}_{2}^{2}}{4 l_{B 2}^{2}}+\frac{\epsilon z_{1}^{*} z_{2}}{2 l_{B 1} l_{B 2}}\right) .
$$

Using Eq. (10), the probability distribution can be presented in the following form

$$
\begin{aligned}
\left|\left\langle\mathbf{r}_{1}, \mathbf{r}_{2} \mid \tilde{0}\right\rangle\right|^{2} & \sim \frac{1}{\sigma_{+} \sigma_{-}} \exp \left(-\frac{\boldsymbol{\rho}_{+}^{2}}{\sigma_{+}^{2}}-\frac{\boldsymbol{\rho}_{-}^{2}}{\sigma_{-}^{2}}\right), \\
\boldsymbol{\rho}_{ \pm} & =\frac{\mathbf{r}_{1}}{l_{B 1}} \pm \frac{\mathbf{r}_{2}}{l_{B 2}}
\end{aligned}
$$

where $\sigma_{ \pm}^{2}=4 /(1 \mp \epsilon)$. This shows that in the new vacuum state $|\tilde{0}\rangle$ the distribution probability for the difference coordinate $\boldsymbol{\rho}_{-}$is squeezed at the expense of the sum coordinate $\boldsymbol{\rho}_{+}$, see Fig. 2 . for the $x$-components of coordinates $\boldsymbol{\rho}_{+}$and $\boldsymbol{\rho}_{-}\left(\right.$at $\left.y_{+}=y_{-}=0\right)$ for several different values of the charge ratio $q_{2} / q_{1}$. Note that in the limit $q_{2} \rightarrow q_{1}\left(\epsilon \rightarrow 1^{-0}\right)$ the relative coordinate distribution becomes maximally squeezed while the center-of-charge coordinate becomes extended, $\sigma_{+} \rightarrow \infty$. This is because the components of $\boldsymbol{\rho}_{1}$ and $\boldsymbol{\rho}_{2}$ do not commute in the lowest LL approximation, see Sec. III below.

\section{NEUTRAL $e-h$ SYSTEMS}

For a neutral system $q_{2}=-q_{1}=q$ the MT operator is given by $\hat{\mathbf{K}}=-i \hbar \boldsymbol{\nabla}_{1}+-i \hbar \boldsymbol{\nabla}_{2}-q \mathbf{B} \times\left(\mathbf{r}_{1}-\mathbf{r}_{2}\right) / 2 c$. Its components commute $\left[\hat{K}_{x}, \hat{K}_{y}\right]=0$ so that the MT group is abelian. Therefore, the states of a neutral magnetoexciton (MX) can be labeled by the magnetic momentum $\mathbf{K}=\left(K_{x}, K_{y}\right) \stackrel{3.5}{n}$ The ground state in zero LL is a $\mathbf{K}=0$ state, which can be presented as a squeezed two-mode vacuum

$$
|\mathbf{K}=0\rangle=S|0\rangle \quad, \quad S=\exp \left(B_{e}^{\dagger} B_{h}^{\dagger}\right) \quad .
$$

The coordinate representation is given by ${ }^{\underline{3}}$

$$
\left\langle\mathbf{r}_{1} \mathbf{r}_{2} \mid \mathbf{K}=0\right\rangle=\exp \left(-\frac{\mathbf{r}_{1}^{2}+\mathbf{r}_{2}^{2}-2 z_{1}^{*} z_{2}}{4 l_{B}^{2}}\right) .
$$

This is a coherent state of an infinite number of electron and hole states in zero LL $\frac{5}{5}$ A state with a finite momentum $\mathbf{K}=\left(K_{x}, K_{y}\right)$ is given by

$$
\begin{aligned}
|\mathbf{K}\rangle & =\left|K_{x}, K_{y}\right\rangle=S(\mathbf{K})|0\rangle \\
S(\mathbf{K}) & =\exp \left[\left(B_{e}^{\dagger}+\frac{i k_{-}}{\sqrt{2}}\right)\left(B_{h}^{\dagger}+\frac{i k_{+}}{\sqrt{2}}\right)\right]
\end{aligned}
$$

and is a two-mode squeezed displaced vacuum state .9 Expectation value of the relative coordinate $\mathbf{r}=\mathbf{r}_{1}-\mathbf{r}_{2}$ in

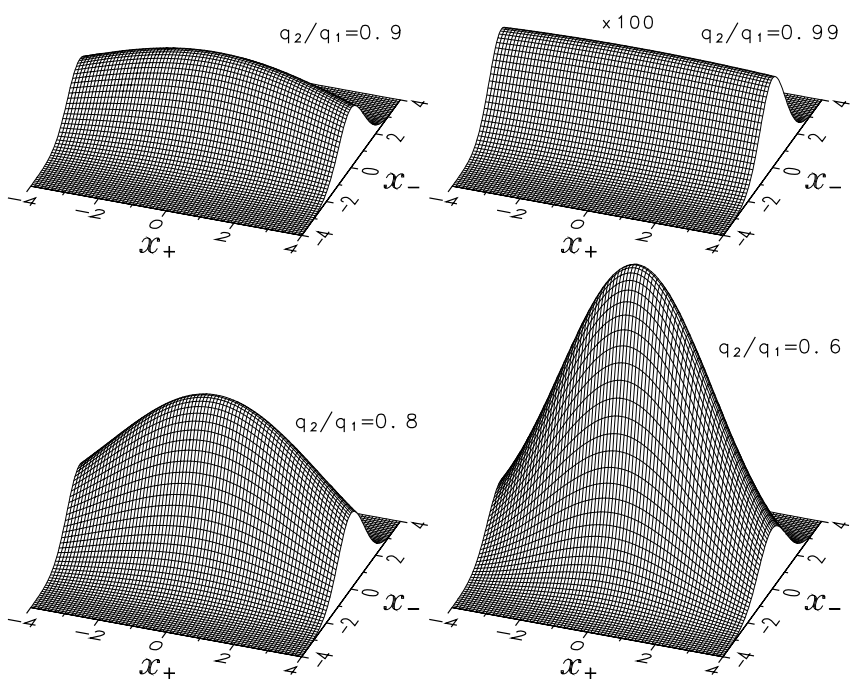

FIG. 2: Probability distribution (11) for the $x$-components of coordinates $\boldsymbol{\rho}_{+}$and $\boldsymbol{\rho}_{-}\left(\right.$at $\left.y_{+}=y_{-}=0\right)$ for several different values of the charge ratio $q_{2} / q_{1}$. 
state (15) is given by $\langle\mathbf{K}|\mathbf{r}| \mathbf{K}\rangle=\frac{\mathbf{B}}{B} \times \mathbf{K} \hbar^{-1} l_{B}^{2}{ }^{3}$. Also, the zero-momentum state (14) can be considered to be a limiting case of a charged system state (10). Indeed, when $q_{2}-q_{1}=0$, wavefunction (10) becomes extended (its norm tends to zero) and its coordinate dependence becomes identical to (14). Using Eq. (11) we deduce that the relative coordinate $\mathbf{r}=\mathbf{r}_{1}-\mathbf{r}_{1}$ becomes maximally squeezed (to the magnetic length $l_{B}=(\hbar c / q B)^{1 / 2}$ in the lowest LL) at the expense of the center-of-charge coordinate $\mathbf{R}=\left(\mathbf{r}_{1}+\mathbf{r}_{1}\right) / 2$. The latter becomes extended. To elucidate this, let us consider projections of the centerof-charge $\overline{\mathbf{R}}$ and relative $\overline{\mathbf{r}}$ coordinates onto zero LL. In complex combinations of the components these are $\bar{X}+i \bar{Y}=\left(B_{e}+B_{h}^{\dagger}\right) / \sqrt{2}$ and $\bar{x}+i \bar{y}=\sqrt{2}\left(B_{e}-B_{h}^{\dagger}\right)=i \hat{K}_{+}$, respectively; here $\hat{K}_{+}=\hat{K}_{x}+i \hat{K}_{y}$. We see that (i) the projected relative and center-of-charge coordinates become canonically conjugate, $[\bar{x}, \bar{Y}]=-[\bar{y}, \bar{X}]=i$, and (ii) the projected relative coordinate, up to a scaling factor and rotation by ninety degrees, coincides with the MT operator $\hat{\mathbf{K}}$.

Let us discuss the relevant two-mode realization of the $S U(1,1)$ generators

$$
\begin{aligned}
& \mathcal{K}_{0}=\frac{1}{2}\left(B_{e}^{\dagger} B_{e}+B_{h}^{\dagger} B_{h}+1\right) \\
& \mathcal{K}_{-}=B_{e} B_{h} \quad, \quad \mathcal{K}_{+}=B_{e}^{\dagger} B_{h}^{\dagger},
\end{aligned}
$$

which satisfy the $S U(1,1)$ commutation relations $\left[\mathcal{K}_{0}, \mathcal{K}_{ \pm}\right]= \pm \mathcal{K}_{ \pm}$and $\left[\mathcal{K}_{-}, \mathcal{K}_{+}\right]=2 \mathcal{K}_{0}{ }^{\underline{6}}$ The dimensionless MT operator for a neutral magnetoexciton, $\hat{\mathbf{k}}^{2}=$ $\hat{\mathbf{K}}^{2} l_{B}^{2} / \hbar^{2}$, becomes in this representation $\hat{\mathbf{k}}^{2}=2\left(2 \mathcal{K}_{0}-\right.$ $\mathcal{K}_{+}-\mathcal{K}_{-}$), while the angular momentum projection $\hat{L}_{z}=$ $B_{h}^{\dagger} B_{h}-B_{e}^{\dagger} B_{e}$ is connected with the Casimir operator of the $S U(1,1) \operatorname{group}^{\underline{6}}$ as $\hat{C}=\left(\hat{L}_{z}^{2}-1\right) / 4$. This allows one to identify states (13) and (15) as generalized coherent states of the $S U(1,1)$ group $\underline{\underline{6}}$ Also, it becomes possible to find a representation of the MX states in the set of $\mathbf{K}^{2}$ and $M_{z}$ quantum numbers, the eigenvalues of the mutually commuting integrals of the motion $\hat{\mathbf{K}}^{2}$ and $\hat{L}_{z}$. The corresponding state for, e.g., $M_{z}=-M \leq 0$, is given by

$$
\left|\mathbf{K}^{2}, M_{z}\right\rangle=e^{B_{e}^{\dagger} B_{h}^{\dagger}}|K ; M\rangle
$$

where

$$
|K ; M\rangle=\frac{1}{\sqrt{I_{M}\left(k^{2}\right)}} \sum_{m=0}^{\infty} \frac{\left(\frac{i k_{-}}{\sqrt{2}}\right)^{m+M}\left(\frac{i k_{+}}{\sqrt{2}}\right)^{m}}{\sqrt{(m+M) ! m !}}|m+M, m\rangle
$$

is the Barut-Girardello coherent state, ${ }^{7} I_{M}(x)$ being a modified Bessel function and $k_{ \pm}=\left(K_{x} \pm i K_{y}\right) l_{B} / \hbar$. Proof follows from Eqs. (17) and the algebra

$$
\mathcal{K}_{-} e^{-\mathcal{K}_{+}}=e^{-\mathcal{K}_{+}}\left(\mathcal{K}_{+}+\mathcal{K}_{-}-2 \mathcal{K}_{0}\right) \quad .
$$

In conclusion, we introduced an operator formalism for partial separation of degrees of freedom for electronhole complexes in magnetic fields. We also established its connection with the $S U(1,1)$ algebras. Application of the powerful formalism of the $S U(1,1)$ group allows one to construct a number of coherent ans squeezed states that may be useful for considering Coulomb correlations and optics of few- and many-particle systems in Landau levels.

This work is supported in part by NSF grants DMR0203560 and DMR-0224225, and by an award of Cottrell Research Corporation.
* Electronic address: adzyubenko@csub.edu URL: http://www.csub.edu/ ${ }^{\sim}$ adzyubenko

1 J. E. Avron, I. W. Herbst, and B. Simon, Ann. Phys. N.Y. 114, 431 (1978).

2 A. B. Dzyubenko, Phys. Rev. B65, 035318 (2001).

3 I. V. Lerner and Yu. E. Lozovik, JETP 51, 588 (1980).

4 M. O. Scully and M. S. Zubairy, Quantum Optics (Cambridge University Press, Cambridge, 1997).

${ }^{5}$ R. B. Laughlin, Physica B126, 254 (1984).

6 A. Perelomov, Generalized Coherent States and Their Ap- plications (Springer-Verlag, Berlin, 1986).

7 A. O. Barut and L. Girardello, Commun. Math. Phys. 21, 41 (1971).

${ }^{8}$ For a multiparticle $e-h$ system, charges $-q_{1}, q_{2}$ and coordinates $\mathbf{r}_{1}, \mathbf{r}_{2}$ correspond to the total charges and center-ofcharge coordinates of the $e$ - and $h$ - subsystems. ${ }^{2}$

${ }^{9}$ Note that for, e.g., the electron operator, $D_{e}(\alpha) B_{e}^{\dagger} D_{e}^{\dagger}(\alpha)=$ $B_{e}^{\dagger}+\alpha$, where a well known displacement operator ${ }^{4.6}$ is given by $D_{e}(\alpha)=\exp \left(\alpha B_{e}^{\dagger}-\alpha^{*} B_{e}\right)$. 